\title{
An Improved Full-Aperture ScanSAR Imaging Method Integrating the MIAA Based Aperture Interpolation
}

\author{
Jiaqi Ning, ${ }^{1,2}$ Dacheng Liu, ${ }^{1}$ Kaiyu Liu, ${ }^{1}$ Heng Zhang $\mathbb{D}^{1},{ }^{1}$ and Yingjie Wang ${ }^{1}$ \\ ${ }^{1}$ Aerospace Information Research Institute, Chinese Academy of Sciences, Beijing, China 100190 \\ ${ }^{2}$ University of Chinese Academy of Sciences, School of Electronic, Electrical and Communication Engineering, Beijing, China 100049 \\ Correspondence should be addressed to Heng Zhang; caszhmail@163.com
}

Received 4 February 2020; Revised 25 May 2020; Accepted 8 June 2020; Published 17 July 2020

Academic Editor: Sang-Hoon Hong

Copyright (c) 2020 Jiaqi Ning et al. This is an open access article distributed under the Creative Commons Attribution License, which permits unrestricted use, distribution, and reproduction in any medium, provided the original work is properly cited.

In the scanning synthetic aperture radar (ScanSAR) mode, the radar antenna sweeps through different range subswaths to image a wide swath. The full-aperture imaging algorithm for ScanSAR data has been widely used because it can be realized by exploiting the existing standard high-precision Stripmap SAR processor and does not require stitch processing in the azimuth. However, both the focused image and the interferogram achieved by full-aperture processing suffer from spikes. The spikes adversely affect the ScanSAR-related applications, such as target detection and interferometry. To effectively suppress the spikes, an improved algorithm based on the missing-data iterative adaptive approach (MIAA) is proposed in this manuscript. Besides, the proposed method can also improve the azimuth resolution of ScanSAR images. Simulation and experimental results demonstrate that this algorithm has better performance when processing ScanSAR data compared with existing methods.

\section{Introduction}

Scanning synthetic aperture radar (ScanSAR) is a kind of operation widely used mode in the modern SAR system [1, $2]$. By periodically sweeping the antenna beam through different range subswaths, ScanSAR can obtain a wide swath at the expense of sacrificing azimuth resolution [3-6]. In each subswath, the received data are blocked into bursts of radar echoes in the azimuth direction. In general, burst-byburst [7-9] and full-aperture [10,11] approaches can be used to process ScanSAR data and these two kinds of approaches have different processing flows. Among them, the burst-by-burst approach is the standard approach, which is computationally efficient. However, the full-aperture approach has also been used a lot by the SAR community now because it can make use of existing standard Stripmap SAR processing programs $[10,12]$. The ScanSAR image focused by the full-aperture approach has been successfully used for lots of applications [13-21]. Particularly, for the ScanSAR data of the ALOS-2 satellite, only the image processed by the full-aperture approach can be used for interferometric applications [13].
In the full-aperture approach, gaps between bursts are filled with zeros and then all the bursts of a subswath can be coherently processed as Stripmap data. However, the coherently processed multiple bursts result in strong interference modulations of the azimuth impulse response function, which are called "spikes" $[5,10,11,22,23]$. These spikes can contaminate ScanSAR image and influence the interpretation. Furthermore, spikes also exist in the phase of the processed ScanSAR images. Thus, the spikes also occur in the ScanSAR interferogram, introducing additional noise to interferogram and degrading the interferogram quality $[5,11,22,23]$. Spike contamination of full-aperture ScanSAR image limits ScanSAR-related applications, such as target detection and interferometry. Therefore, the development of effective techniques to suppress the spikes is very useful. Although the spikes can be reduced by a low-pass filtering operation [10], the residual effect is enough to cause image visual disturbances [22], and the originally rough azimuth resolution will get worse by this approach [10]. As the azimuth resolution of the full-aperture result is controlled by the envelope of the spikes [10], the spike suppression can enhance the azimuth resolution at the same time. 


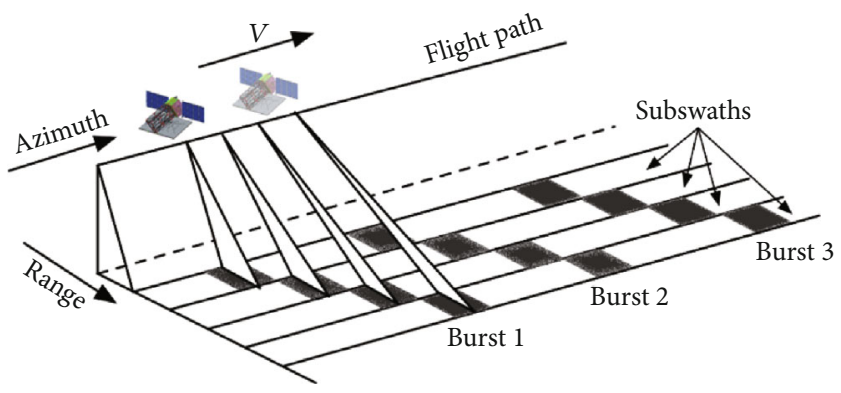

(a)

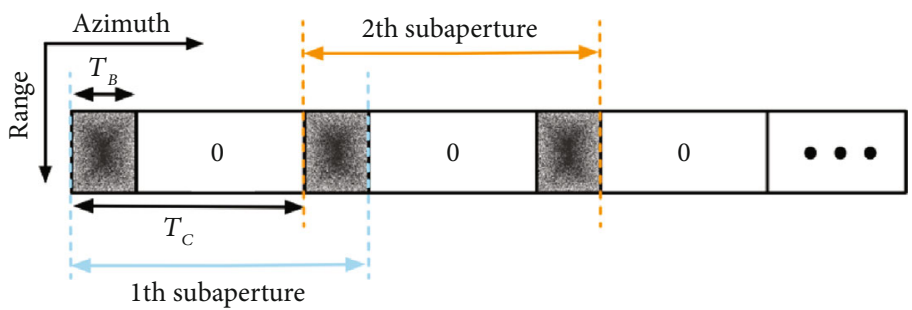

(b)

Figure 1: (a) The ScanSAR operation mode. (b) Acquired signal of a ScanSAR subswath.

The algorithms of suppressing the spikes while improving azimuth resolution are very beneficial to the ScanSARrelated applications.

In [22], an algorithm referred to as the linear prediction model aperture interpolation technique (LPM-AIT) was proposed to suppress spikes when the full-aperture imaging algorithm is used to process ScanSAR data. In the LPMAIT, the gaps between bursts are recovered by the linear prediction model (LPM), and the coefficient of the LPM is estimated by the Burg algorithm [24]. A similar method is also used in the data infilling part of the algorithm in [8] to enhance the azimuth resolution when ScanSAR data imaged by a burst-by-burst approach. However, when using the LPM, the missing-data proportion should be no more than $50 \%$ [25], and the data recovery capability of the LPM decreases significantly as the proportion of missing data increases, which makes this algorithm only suitable for the ScanSAR system with no more than 2 subswaths. The number of subswaths in the ScanSAR mode is typically 2-5 [10], which means that the missing-data proportion will reach $50 \%$ to $80 \%$. Thus, the spike suppression performance of the LPM-AIT is limited when the ScanSAR data are processed by the full-aperture imaging algorithm.

In this manuscript, an improved full-aperture ScanSAR imaging algorithm integrating the missing-data iterative adaptive approach (MIAA) based aperture interpolation technique (MIAA-AIT) is proposed. The remainder of this manuscript is organized as follows. The problem we studied is described in Section 2. Section 3 is a description of the proposed method. The results and discussions of the method are given in Section 4 , and the conclusions are drawn in Section 5.

\section{Problem Statement}

ScanSAR obtains a wide-swath image by periodically sweeping the antenna beam through different range subswaths, as shown in Figure 1(a). In each subswath, the received signal in the range direction is the same as those in the Stripmap mode. In the azimuth direction, however, the received signal is blocked into bursts and is equivalent to data truncated from the Stripmap mode data which causes incomplete signal phase history. Two adjacent bursts and zeros between them as a whole is called a subaperture, as shown in Figure 1(b).

With $T_{B}$ as the burst duration and $T_{C}$ as the burst cycle period, $N_{B}=T_{B} \times$ PRF is the number of samples per burst, and $N_{C}=T_{C} \times \mathrm{PRF}$ is the number of samples in a burst cycle period, where PRF is the sampling frequency in the azimuth direction. Setting the number of subswaths in the ScanSAR system to $N_{S}$, then $T_{C}=N_{S} \times T_{B}[7,26,27]$. The gaps between bursts can be treated as missing samples. The missing proportion $\gamma$ can be expressed as (1)

$$
\gamma=\frac{T_{C}-T_{B}}{T_{C}}=\frac{N_{C}-N_{B}}{N_{C}}=1-\frac{1}{N_{S}} \# .
$$

In the following, we focus only on the azimuth direction of one subswath. For ScanSAR mode, a point-target azimuth signal before azimuth compression is

$$
S_{\text {raw }}(\eta)=\sum_{n} A_{0} \cdot \operatorname{rect}\left(\frac{\eta-\eta_{c}^{n}}{T_{B}}\right) \exp \left\{j \pi K_{a} \eta^{2}\right\} \#,
$$

where $\eta$ is the azimuth time, $A_{0}$ is an arbitrary complex constant, $K_{a}$ is the azimuth chirp rate, $n$ is the burst number, $\eta_{c}$ is the burst center, and the zero Doppler moment of the target $\eta_{d}$ is set as zero. After padding zeros in the burst intervals, ScanSAR mode data can be coherently processed by standard Stripmap processors, referred to as the full-aperture approach. A point response of ScanSAR using the full-aperture imaging algorithm is equivalent to coherently adding the compression results of each burst. 


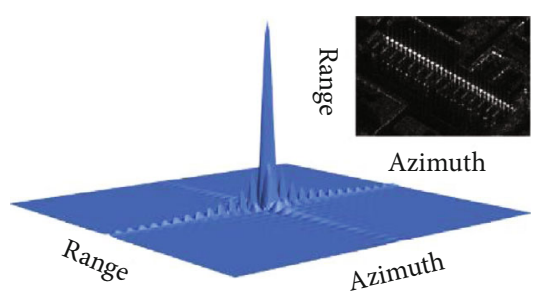

(a)

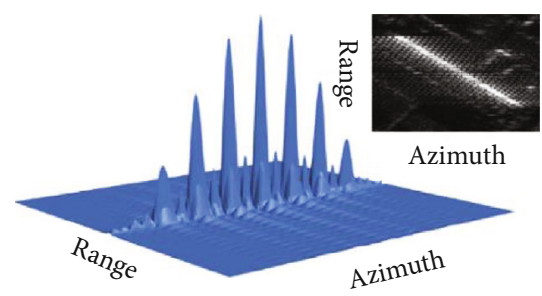

(b)

FIGURE 2: Schematic facade in two dimensions of a point target in Stripmap mode and ScanSAR mode. The upper right corner shows a real SAR scene. (a) Stripmap mode. (b) ScanSAR mode.

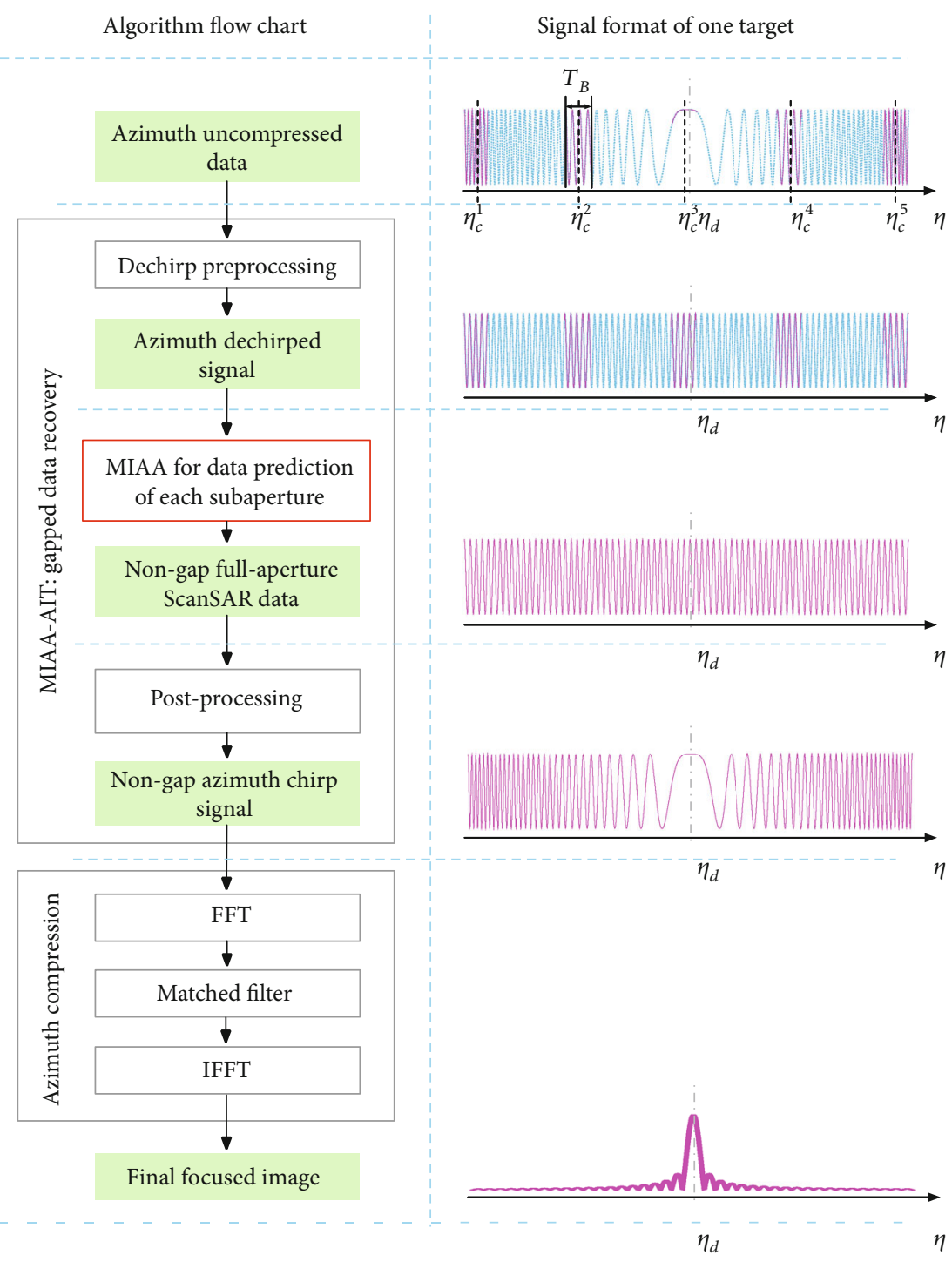

FIGURE 3: The diagram of processing flow chart and schematic signal format of one target in each step.

$$
S(\eta)=\sum_{n} A_{0} T_{B} \operatorname{sinc}\left(K_{a} T_{B} \eta\right) \exp \left\{j \pi K_{a} \eta\left(\eta-2 \eta_{c}^{n}\right)\right\} \#
$$

When several such pulses are added together, different phase slopes give spikes in the summation output. As shown in Figure 2(b), the main lobe and side lobe of the two- dimensional focus result of one point target are split into several parts along the azimuth direction, which is called "spike" phenomenon. Compared to the point target of the Stripmap mode, shown in Figure 2(a), the focus result of the point target is strongly deteriorated due to the existence of spikes. The upper right corner of Figures 2(a) and 2(b) are 


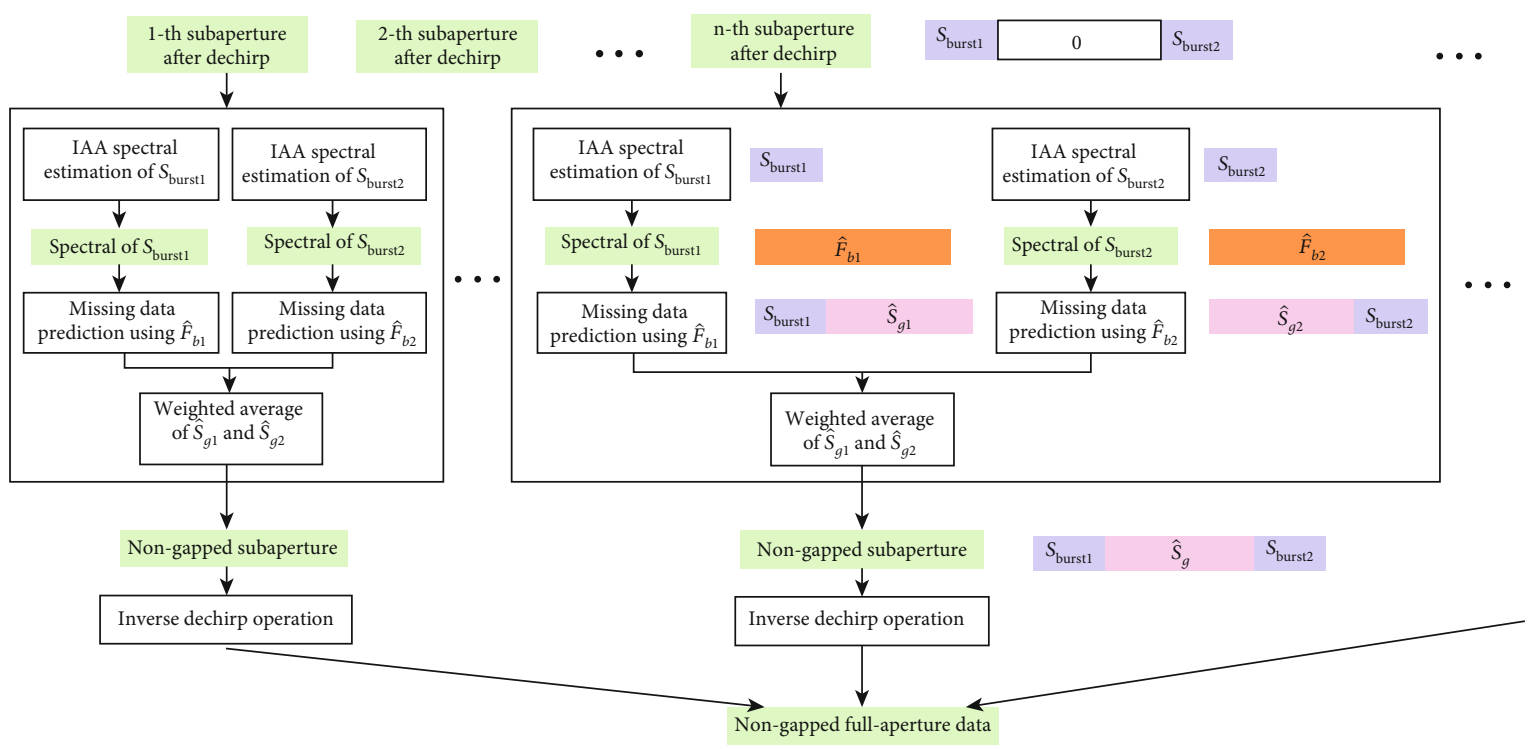

Figure 4: Processing flow of the MIAA-AIT.

the images of strip and ScanSAR mode in the same real SAR scence, respectively. We can see that the spikes seriously contaminate the ScanSAR image and influence the interpretation and applications [22].

Infilling the gaps between bursts with valid data is an effective strategy to suppress spikes and improve the azimuth resolution at the same time. Li et. al [22] used the LPM for data infilling between the burst. However, the number of samples that can be effectively predicted by the LPM is limited, and the missing-data proportion should be no more than $50 \%$ [25]. The number of subswaths in the ScanSAR mode is typically 2-5 [10], which means that the missingdata proportion will reach $50 \%$ to $80 \%$. The performance of the LPM degrades significantly, and the effect of spike suppression is limited at such a high missing-data proportion. Therefore, a spike suppression approach that is more suitable for the ScanSAR data should be proposed.

\section{Proposed MIAA-AIT Algorithm}

After range compression and range cell migration correction (RCMC), we aim at recovering the gaps of the azimuth uncompressed ScanSAR data by the MIAA-AIT algorithm. Recovering missing data caused by incomplete phase history from available data is widely used in $[24,25]$. To make the recovered result more reliable, the quadratic phase term is usually removed by the dechirp operation and retained the linear phase term [22]. Because the phase history in ScanSAR mode is limited to the short span of the image by the antenna beam width, performing dechirp operation on the entire data will cause a large number of targets located at the same distance bin to have the same frequency, which will cause target confusion [9]. Thus, the proposed MIAA-AIT is performed for each subaperture to obtain nongapped subswath data.

Figure 3 shows the diagram of the processing flow chart and schematic signal format of one target in each step. The green boxes represent signal formats at different steps. The proposed MIAA-AIT includes three steps which are dechirp operation, MIAA for missing data prediction, and postprocessing. Among them, the red square is the key step of the manuscript, which will be explained in detail in the following. Finally, the azimuth compression is applied to the nongapped subswath data to acquire the final focused ScanSAR image.

3.1. Dechirp Preprocessing. The phase of each target is quadratic, as shown in (2), which is inconvenient for the application of MIAA. Dechirp preprocessing in the azimuth is performed by multiplying the conjugate of the azimuth chirp for each subaperture. After the dechirp processing, the target phase is linear with the azimuth, which is suitable for applying the MIAA.

3.2. Gap Recovery by the MIAA. The detailed data recovery process for each subaperture is shown in the black box of Figure 4. Let us take the n-th single subaperture as an example. Supposing that (4) is the azimuth signal of a subaperture after dechirp preprocessing,

$$
\boldsymbol{S}_{\boldsymbol{s}}=\left[\boldsymbol{S}_{\text {burst } 1\left[1 \times N_{B}\right]} \boldsymbol{S}_{\mathrm{gap}\left[1 \times\left(N_{C}-N_{B}\right)\right]} \boldsymbol{S}_{\text {burst } 2\left[1 \times N_{B}\right]}\right]^{T} \#,
$$

where $[\cdot]^{T}$ denotes the transpose, $S_{\text {burst1 }}$ and $S_{\text {burst2 }}$ are the samples of two bursts in a subaperture which are available samples, and $S_{\text {gap }}$ is the missing samples between two bursts that need to be estimated. The MIAA uses iterative adaptive approach (IAA) spectrum estimate [28] to retrieve the missing data. First, the MIAA uses the IAA to obtain an accurate spectral estimation from the given samples. Then, the missing-data recovery step is performed using the IAA spectrum estimates by a linear minimum mean-squared error (MMSE) estimator with a negligible additional computational burden [29]. In the implementation of the proposed MIAA-AIT, the missing samples are recovered by the weighted average of the estimated results by $\boldsymbol{S}_{\text {burst1 }}$ and $S_{\text {burst2 }}$ for robustness and efficiency. 


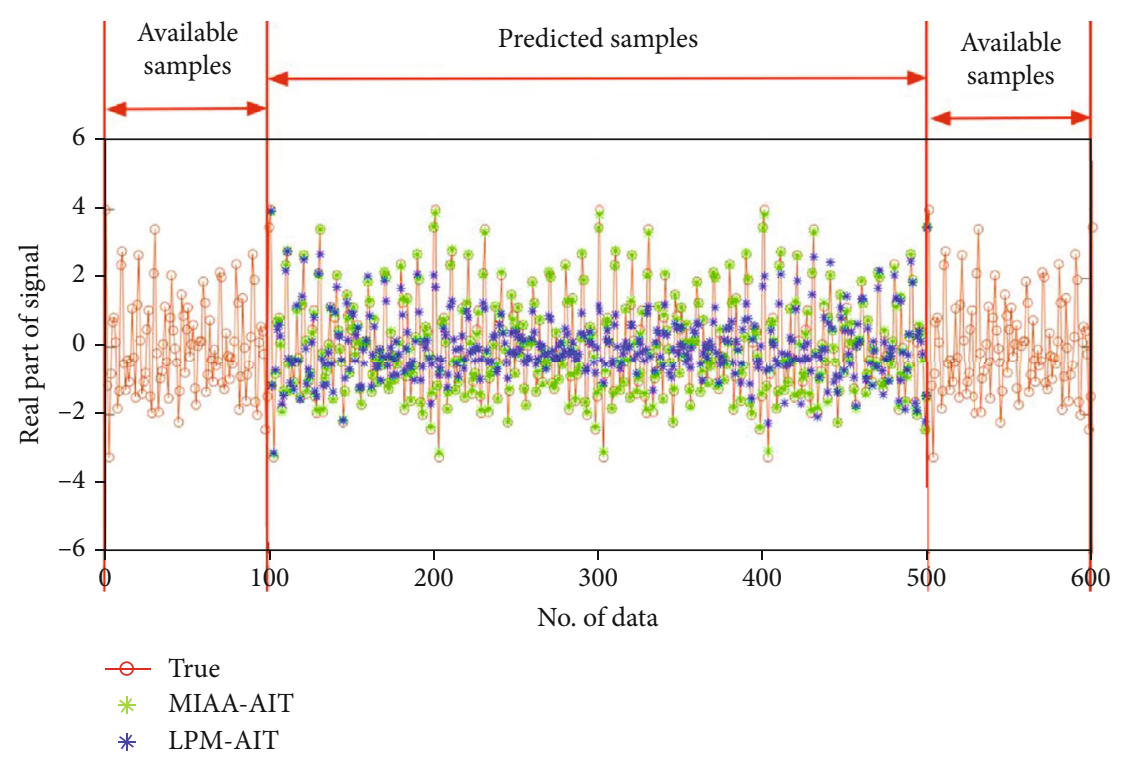

(a)

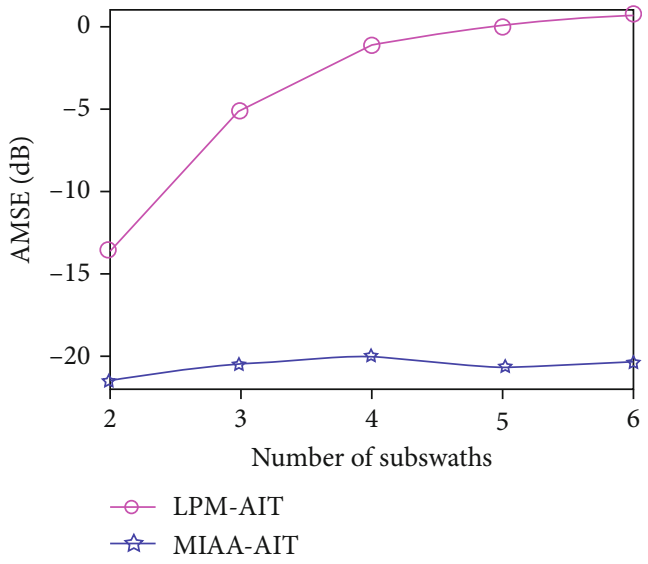

(b)

Figure 5: Comparison of the effectiveness of missing-data recovery. (a) Missing data recovery results for one random trial when the missingdata proportion is $80 \%$. (b) AMSEs of the estimation results for different missing-data proportions.

Let us take the estimation by $S_{\text {burst1 }}$ as an example. Let $\boldsymbol{S}_{b}=\boldsymbol{S}_{\text {burst } 1}, \boldsymbol{S}_{g}=\boldsymbol{S}_{\text {gap }}$ and $\boldsymbol{S}=\left[\boldsymbol{S}_{b} \boldsymbol{S}_{g}\right]^{T}$. Let $\omega_{k}=2 \pi(k / K), k=$ $0,1,2 \cdots K-1$ be the frequency points in the frequency domain. $K$ should be much larger than $N_{B}[28,29]$, and $K$ is set to 8 times of $N_{B}$ in our experiments. Let

$$
\begin{aligned}
\boldsymbol{a}_{b}\left(\omega_{k}\right) & =\left[1 e^{j \omega_{k}} \cdots e^{j\left(N_{B}-1\right) \omega_{k}}\right]^{T} ; \boldsymbol{a}_{g}\left(\omega_{k}\right) \\
& =\left[e^{j N_{B} \omega_{k}} e^{j\left(N_{B}+1\right) \omega_{k}} \cdots e^{j\left(N_{C}-1\right) \omega_{k}}\right]^{T} \#,
\end{aligned}
$$

where $\boldsymbol{a}_{b}\left(\omega_{k}\right)$ and $\boldsymbol{a}_{g}\left(\omega_{k}\right)$ are the Fourier vectors corresponding to $S_{b}$ and $S_{g}$ at frequency $\omega_{k}$.

First, the spectral of the available data $S_{b}$ at the frequency $\omega_{k}$ is estimated by the IAA, referred to as $\widehat{F}_{b 1}\left(\omega_{k}\right)$. The IAA makes use of an adaptive weighting matrix to get the spectral of the available data in an iterative manner until convergence.

$$
\widehat{F}_{b 1}\left(\omega_{k}\right)=\frac{\boldsymbol{a}_{b}^{H}\left(\omega_{k}\right) \boldsymbol{R}^{-1} \boldsymbol{S}_{b}}{\boldsymbol{a}_{b}^{H}\left(\omega_{k}\right) \boldsymbol{R}^{-1} \boldsymbol{a}_{b}\left(\omega_{k}\right)} \#,
$$

where $(\cdot)^{H}$ denotes the conjugate transpose and $\boldsymbol{R}$ is the covariance matrix of the given data, i.e.,

$$
\boldsymbol{R}=\sum_{k=0}^{K-1}\left|F \wedge_{b 1}\left(\omega_{k}\right)\right|^{2} \boldsymbol{a}_{b}\left(\omega_{k}\right) \boldsymbol{a}_{b}^{H}\left(\omega_{k}\right) \#
$$

The initialization of the IAA can be completed by setting $\boldsymbol{R}$ to the identity matrix $\boldsymbol{I}$. Instead of computing $\boldsymbol{R}$, we can directly get the inverse of $\boldsymbol{R}$ in each iteration. By taking advantage of the Toeplitz structure of the covariance matrix, the inversion of the covariance matrix can be converted to matrix multiplication operations with a much lower calculation amount $[30,31]$. Typically, no more than 10 to 15 iterations are required for (6) reaching convergence [28, 29]. 


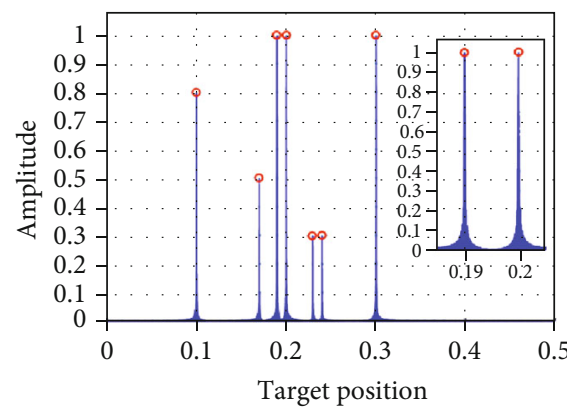

(a)

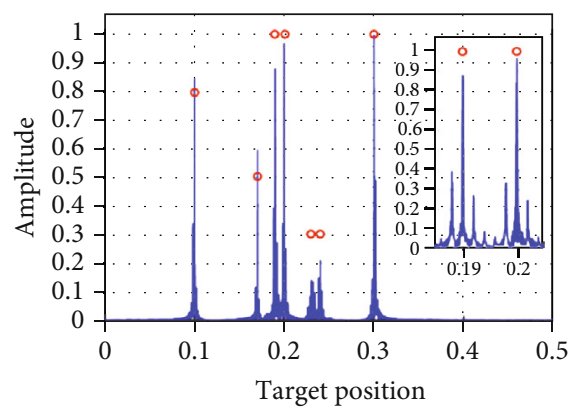

(c)

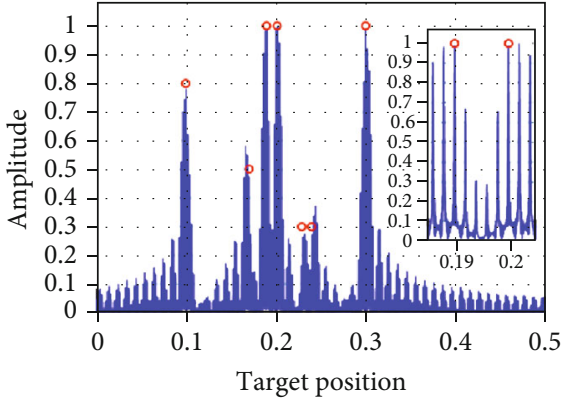

(b)

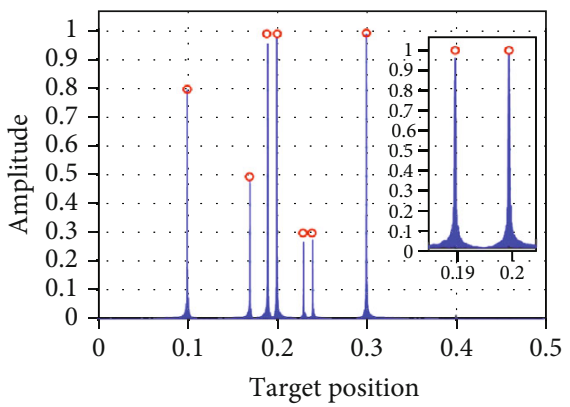

(d)

Figure 6: Comparison of target amplitude estimation results with (a) complete samples. (b) Available samples. (c) Available samples and LPM-AIT-estimated gap samples. (d) Available samples and MIAA-AIT-estimated gap samples.

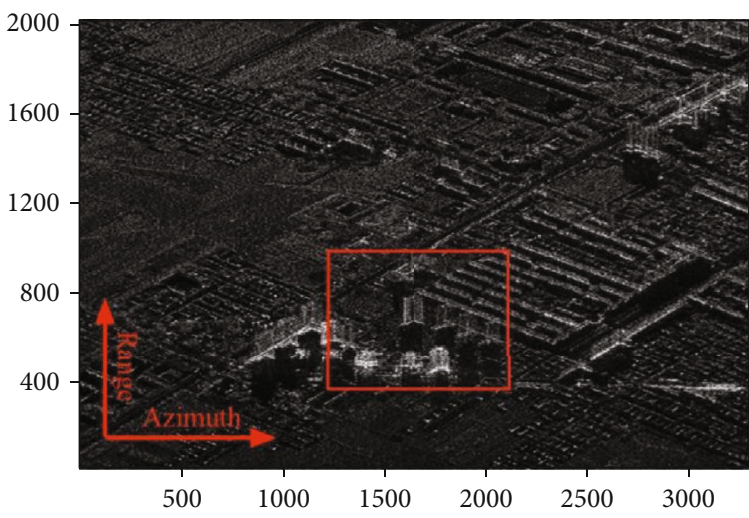

(a)

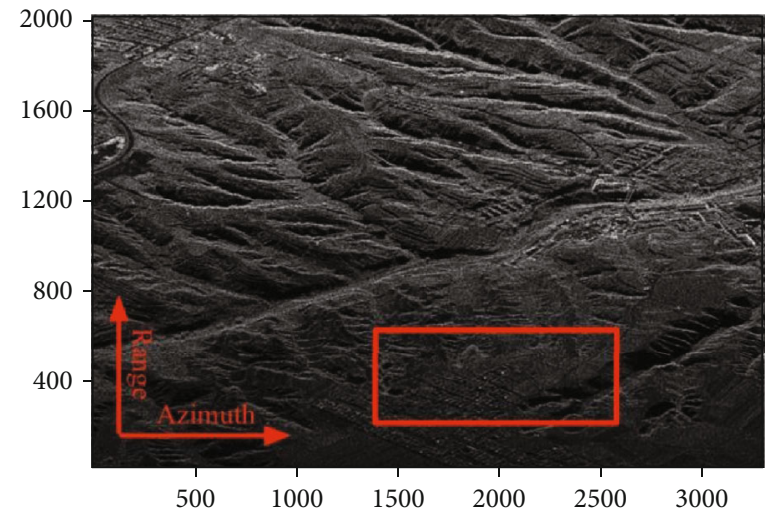

(b)

FIgURE 7: Two representative scenes imaged by the proposed algorithm. (a) Urban areas. (b) Rural areas.

Once the estimated spectrum $\widehat{F}_{b 1}\left(\omega_{k}\right)$ has been computed, the missing samples in the time domain can be estimated by an MMSE estimator as shown in (8), which is the second step of the MIAA [29].

$$
\widehat{\boldsymbol{S}}_{g 1}=\sum_{k=0}^{K-1}\left[\left|F \wedge_{b 1}\left(\omega_{k}\right)\right|^{2} \boldsymbol{a}_{b}^{H}\left(\omega_{k}\right) R^{-1} \boldsymbol{S}_{b}\right] \boldsymbol{a}_{g}\left(\omega_{k}\right) \#,
$$

Note that $\widehat{F}_{b 1}\left(\omega_{k}\right)$ can be obtained from (6), and $\boldsymbol{a}_{b}^{H}\left(\omega_{k}\right)$ $R^{-1} S_{b}$ is the numerator on the right side of (6), which suggests that the computation cost of the missing data estimation after the IAA is very low. The whole missing samples estimation processing is parameter free. Similarly, the processing of obtaining $\widehat{\boldsymbol{S}}_{g 2}$ with $\boldsymbol{S}_{\text {burst2 }}$ is similar to obtaining
$\widehat{\boldsymbol{S}}_{g 1}$ with $\boldsymbol{S}_{\text {burstl }}$ in the same way. Then, the missing samples are recovered by the weighted average of the $\widehat{S}_{g 1}$ and $\widehat{S}_{g 2}$ as shown in Figure 4.

3.3. Postprocessing. The inverse operation of dechirp processing in step 3.1 is applied to recover the subaperture signal format corresponding to the standard ScanSAR mode. Figure 4 shows how to use the proposed MIAA-AIT to recover the gaps between the bursts. After all the gaps are recovered, subapertures are stitched together along the azimuth direction to obtain nongapped full-aperture data. Finally, azimuth compression is completed for nongapped full-aperture data, and the enhanced ScanSAR image can be obtained as shown in Figure 3. 


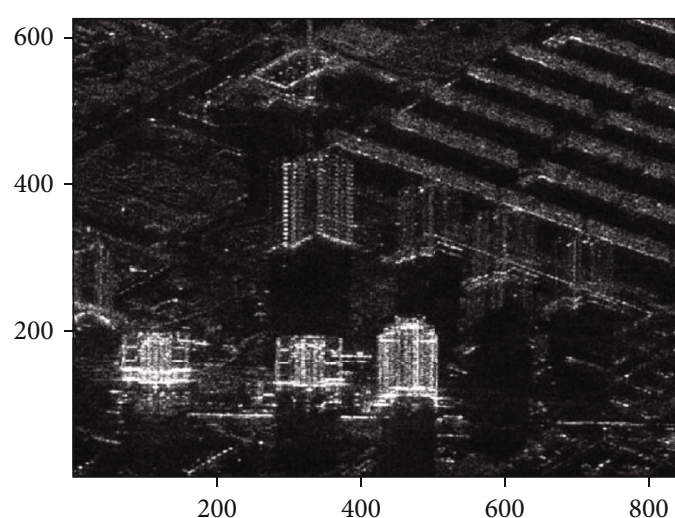

(a)

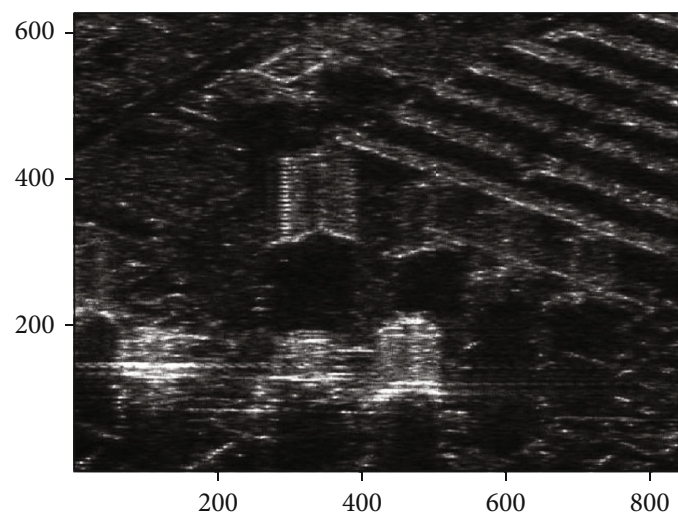

(c)

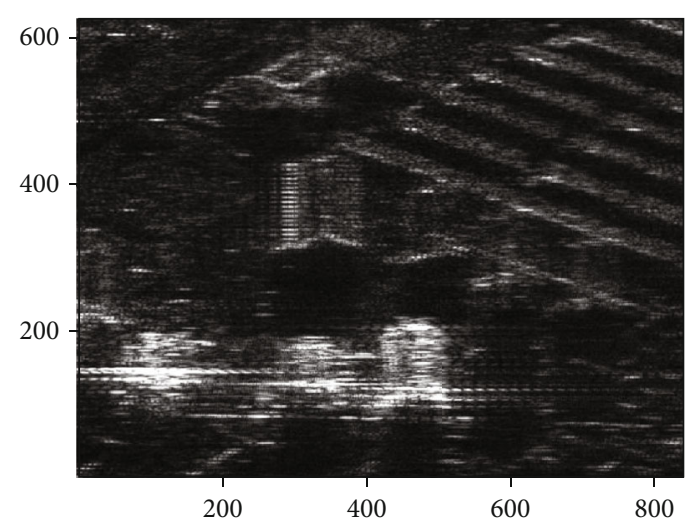

(b)

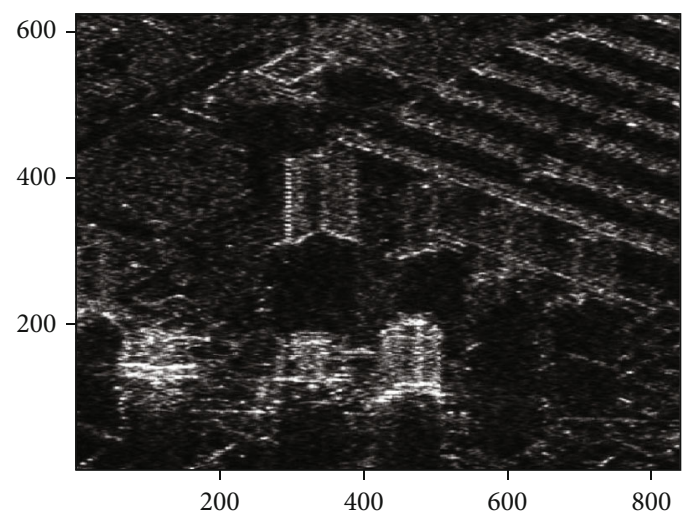

(d)

FIGURE 8: Magnified area shown in Figure 7(a). (a) Stripmap mode. (b) ScanSAR data imaged by the full-aperture approach. (c) ScanSAR data imaged by the LPM-AIT. (d) ScanSAR data imaged by the MIAA-AIT.

\section{Result}

4.1. Simulation Results. Here, one-dimensional (1-D) simulation results are presented to demonstrate the performance of the proposed MIAA-AIT in two aspects: missing data recovery and spike suppression.

4.1.1. Accuracy of Missing Sample Estimation. The signal we consider consists of seven complex-valued sinusoidal components located at normalized frequencies $f_{1}=0.1, f_{2}=$ $0.17, f_{3}=0.19, f_{4}=0.2, f_{5}=0.23, f_{6}=0.24$, and $f_{7}=0.3$ with complex amplitudes $A_{1}=0.8, A_{2}=0.5, A_{3}=A_{4}=1, A_{5}=A_{6}$ $=0.3$, and $A_{7}=1$. The phases of this seven targets are set as $\alpha_{1}=0.5, \alpha_{2}=0.3, \alpha_{3}=0.8, \alpha_{4}=0.5, \alpha_{5}=0.6, \alpha_{6}=0$, and $\alpha_{7}$ $=0.8$. The signal is corrupted by Gaussian white noise with a zero mean and a standard deviation of 0.1 . We first illustrate the missing-data recovery ability of the proposed algorithm for the case of an $80 \%$ missing-data proportion, which represents five subswaths. The burst length $N_{B}$ is 100 in the simulations, and therefore, the number of missing data points between two bursts in a subaperture is 400 . The recovery result of the 400 missing samples for one random trial among 100 Monte Carlo trials is presented in Figure 5(a). It can be concluded from Figure 5(a) that all the missing samples can be estimated with high accuracy by the proposed MIAA-AIT algorithm. With the LPM-AIT, however, only

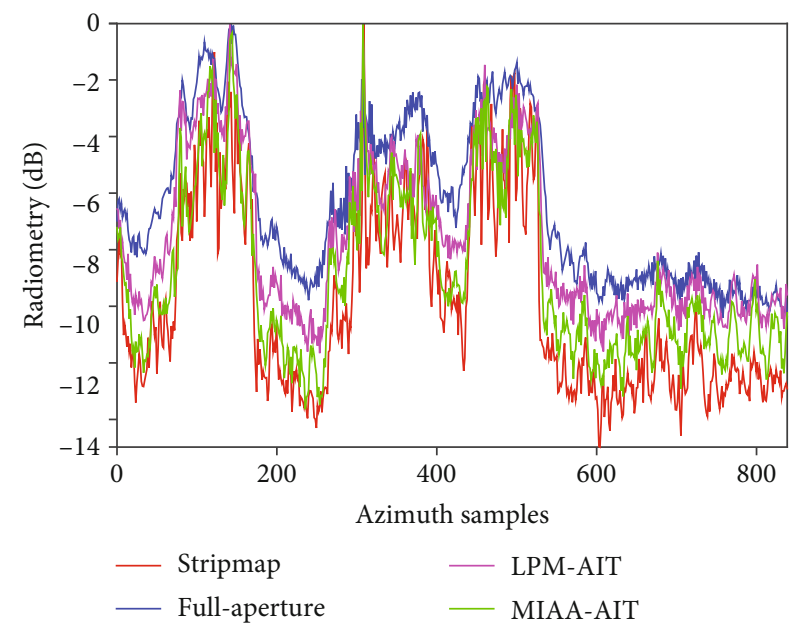

FIGURE 9: Radiometry comparison result of MIAA-AIT, LPM-AIT, full-aperture, and Stripmap over Figure 8.

missing samples that are close to the available data can be estimated with a relatively high accuracy, while the estimates in the middle exhibit a large deviation.

Next, we fix the burst length $N_{B}=100$ and change the missing-data proportion from $50 \%$ to $83.3 \%$, which represents 2 to 6 subswaths. The standard deviation of the 


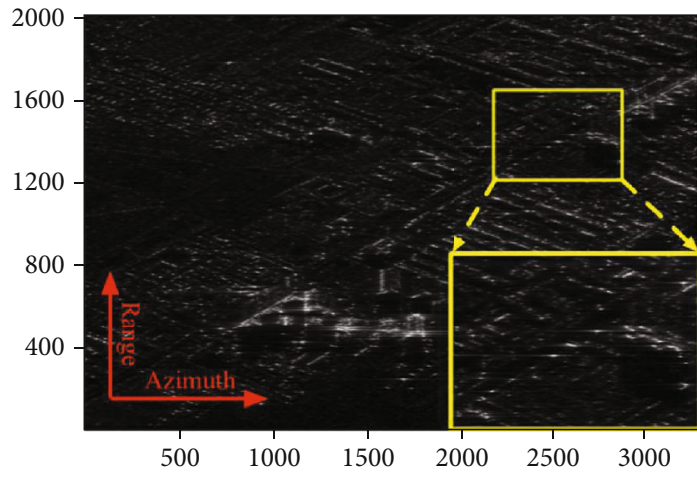

(a)

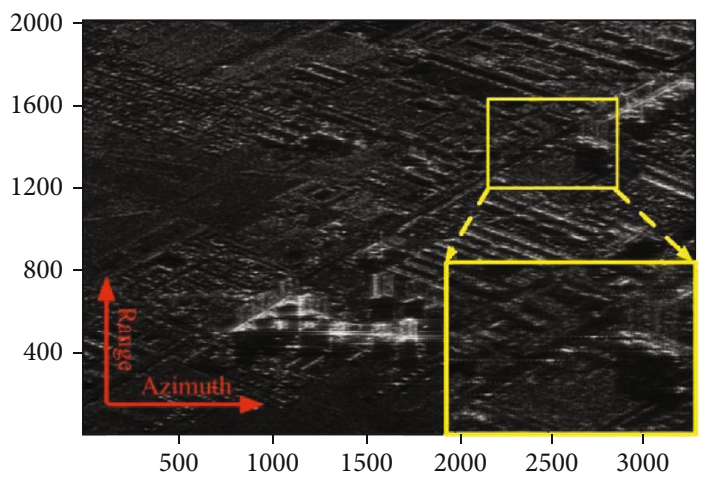

(b)

FIGURE 10: (a) Imaging result using only the data recovered by the LPM-AIT. (b) Imaging result using only the data recovered by the proposed algorithm.

Gaussian white noise is fixed at 0.1 . For each case, 100 Monte Carlo trials are performed. The average mean squared error (AMSE) of the MIAA-AIT and the LPM-AIT for different missing-data proportions are shown in Figure 5(b). It can be concluded that the effect of the proposed algorithm is better than the LPM-AIT as the missing-data proportion increases. And the effect of the LPM-AIT deteriorates significantly when the missing-data proportion exceeds $50 \%$. The average AMSE of MIAA-AIT is $-20.5185 \mathrm{~dB}$. The average AMSE of LPM-AIT is $-7.6171 \mathrm{~dB}$, which is $12.9014 \mathrm{~dB}$ higher than that of MIAA-AIT. These results demonstrate that the MIAA-AIT can predict missing data more accurately than the LPM-AIT. As a result, the proposed algorithm is more practical in the ScanSAR mode.

4.1.2. Performance in Spike Suppression. The effect of suppressing spikes caused by coherently processed multiple bursts is evaluated in this section. In this simulation, ten subapertures are used and the signal and noise settings are the same as that in part 4.1.1. The focused amplitude results of the complete samples, the available samples, the available samples with LPM-AIT-estimated samples, and the available samples with MIAA-AIT-estimated samples are shown in Figures 6(a)-6(d), respectively. The result of processing with gapped samples shows obvious spikes, as shown in Figure 6(b). In comparison, the LPM-AIT has some effect in alleviating the spikes, however, spikes still exist as shown in Figure 6(c). In Figure 6(d), spikes are almost completely suppressed, and the amplitudes are more accurate. Taking the targets amplitude estimation results with complete samples (Figure 6(a)) as reference, we calculate the difference between the other three estimation results and the reference, respectively. The sum of the difference between the amplitude estimation result from available samples (Figure 6(b)) and the reference is 72.898 . The sum of the difference between the amplitude estimation result from LPM-AITestimated samples (Figure 6(c)) and the reference is 14.027. The sum of the difference between the amplitude estimation result from MIAA-estimated samples (Figure 6(d)) with the reference is 1.472 . It can be seen from the numerical comparison that the amplitude estimated results by MIAA-AIT are more similar to the reference, which means the MIAA-AIT is more effective than the LPM-AIT. It is obvious that the MIAA-AIT has a better performance than the LPM-AIT in spike suppression.

4.2. Experimental Results. To demonstrate the performance of the proposed algorithm in spike suppression, experimental results on real SAR data are provided. As described in Section 2 and Figure 2(b), in the range direction, the received raw signal of ScanSAR are the same as those in the Stripmap mode; in the azimuth direction, the raw ScanSAR data are equivalent to the periodically truncated Stripmap mode data. Therefore, obtaining raw data of the ScanSAR mode is equivalent to introducing periodic gaps into raw stripmap data. In addition, to verify the effectiveness of the proposed algorithm in the real SAR scene, we need to utilize the imaging result of the Stripmap mode as a reference. Thus, in our experiment, we obtain ScanSAR mode raw data by introducing periodic gaps into raw stripmap data.

The raw data were collected with an experimental interferometric airborne SAR system developed by the Institute of Electronics, Chinese Academy of Science. The system operates at the C-band $(5.4 \mathrm{GHz})$; the PRF is $750 \mathrm{~Hz}$; the average flight speed is $68 \mathrm{~m} / \mathrm{s}$, and the size of the antenna is $0.9 \mathrm{~m}$. First, the spike suppression performance of the proposed algorithm is demonstrated. Then, interferograms acquired with the MIAA-AIT are given.

4.2.1. The Results of Spike Suppression. In the experiment, the ScanSAR system has five subswaths with a missing-data proportion of $80 \%$, which is generally used in the ScanSAR system. The length of the synthetic aperture time is about 5000 samples, the length of one burst is set as 300 samples, and the length of the burst cycle is 1500 samples. Two representative scenes, shown in Figure 7, are selected to demonstrate the advantages of the proposed algorithm. Both two scenes are corresponding to 11 bursts with an area of 1485 meters in azimuth direction and 1100 meters in range direction. The pixel spacing in the azimuth is about one-fifth of the pixel spacing in range; thus, the images are multilooked 5 times in azimuth when displaying. The overall ScanSAR images are well focused, and no obvious spike phenomena exist. 


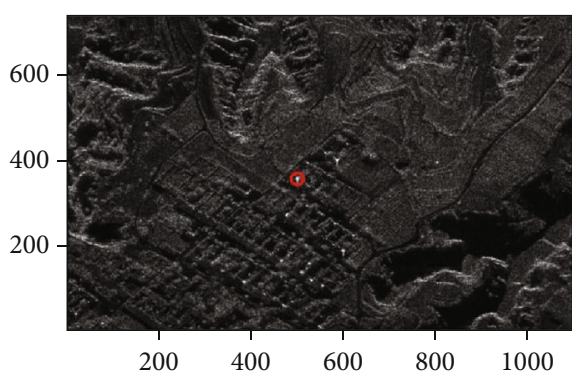

(a)

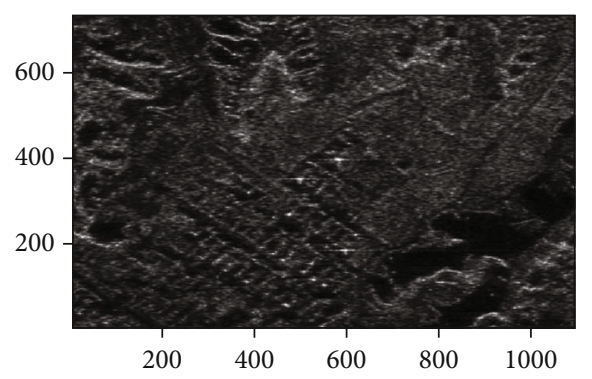

(c)

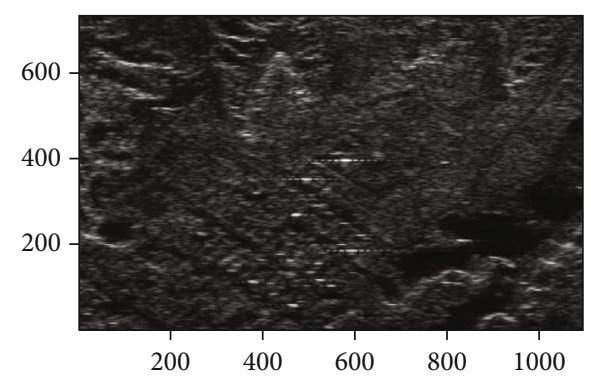

(b)

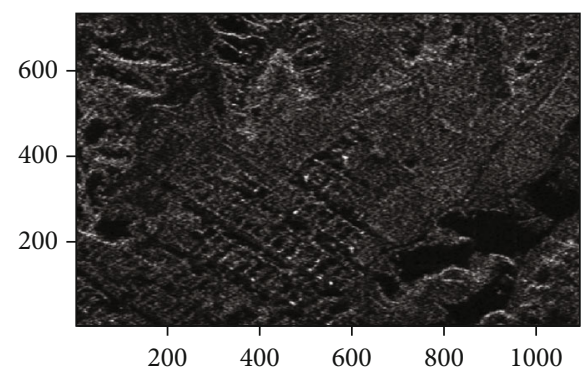

(d)

FIGURE 11: Magnified area shown in Figure 7(b). (a) Stripmap mode. (b) ScanSAR data imaged by a full-aperture approach. (c) ScanSAR data imaged by the LPM-AIT. (d) ScanSAR data imaged by the MIAA-AIT.

The area shown in Figure 7(a) is primarily composed of man-made structures, representing an urban area. The local scene highlighted in Figure 7(a) by a red rectangle is magnified for a comparison with the other algorithms as shown in Figure 8. Based on a visual comparison of Figures $8(\mathrm{~b})-8(\mathrm{~d})$, the LPM-AIT can reduce the spikes to some extent compared to the full-aperture approach. The proposed algorithm has a better performance in spike suppression. The outlines of the artificial buildings are clearer in Figure 8(d) because the spikes are suppressed more thoroughly by the proposed algorithm.

To illustrate the advantage of the proposed method in radiometry performance, we integrate pixels of the amplitude image of Figures 8(a)-8(d) across range direction as shown in Figure 9. In the area of buildings where most of the scatterers are bright scatterers, the performance of both MIAA-AIT and LPM-AIT is close to that of the Stripmap and the performance of the MIAA-AIT is slightly better than the LPM-AIT. This shows that both MIAA-AIT and LPM-AIT have a good processing effect on bright scatterers, but MIAA-AIT is better than LPM-AIT. In the area near the buildings, where few strong scatterers represent, the difference in radiometry performance is relatively large. The radiometry performance in these areas reflects the spike suppression performance of the algorithms. The lower the value, the better the spike suppression performance. Overall, the value of MIAA-AIT is lower than that of the LPM-AIT and closer to the value of the Stripmap. Thus, the MIAA-AIT has better suppression performance than the LPM-AIT. This further demonstrates the image quality improvement of the proposed algorithm.

We want to verify whether this improvement comes from the improvement of the quality of the recovered missing data by the proposed algorithm compared to the LPM-AIT. By

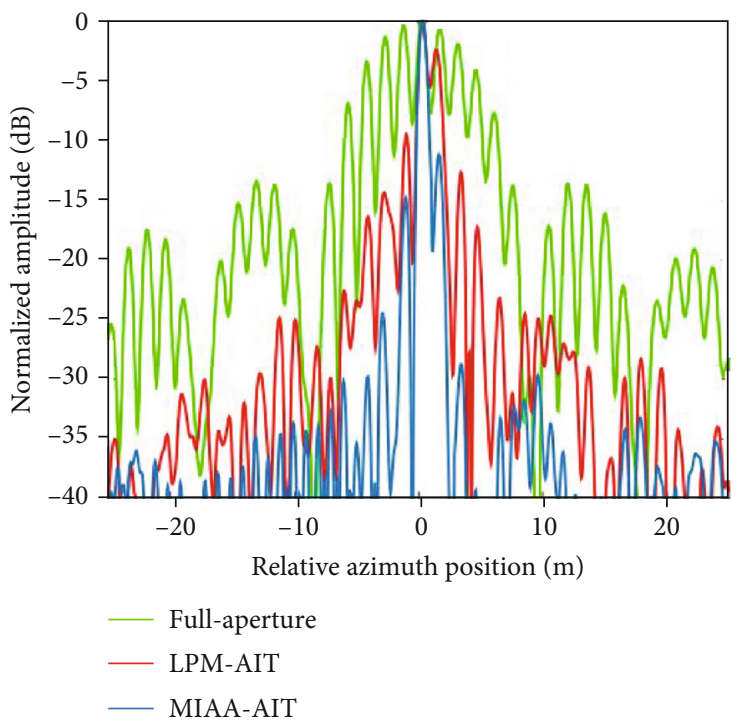

Figure 12: Azimuth profiles of the selected point-like target in Figure 10(a).

TABLE 1: IC comparison of two representative scenes (Figures 8 and 10).

\begin{tabular}{lcc}
\hline & IC value of Figure 8 & IC value of Figure 10 \\
\hline Full-aperture & 2.2061 & 1.0281 \\
LPM-AIT & 2.8176 & 1.2505 \\
MIAA-AIT & 3.1959 & 1.5731 \\
\hline
\end{tabular}




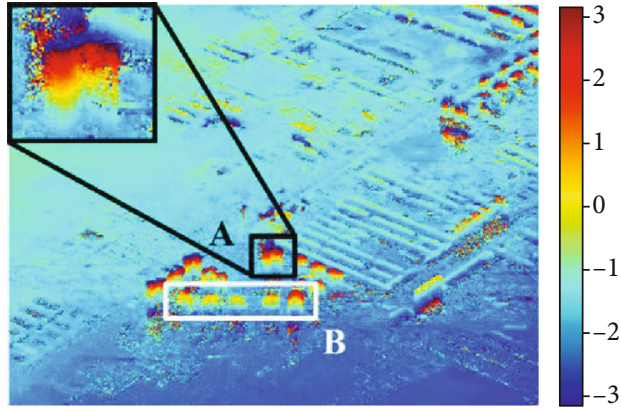

(a)

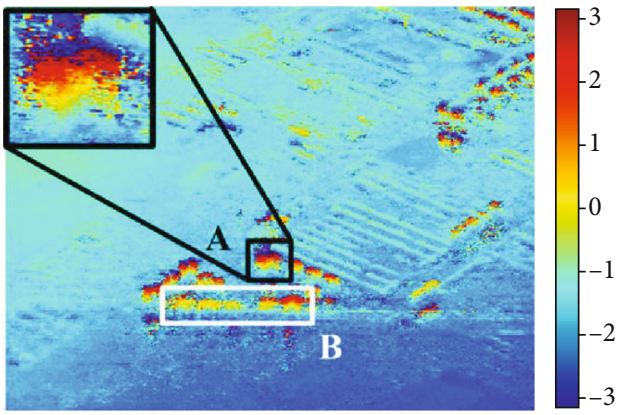

(c)

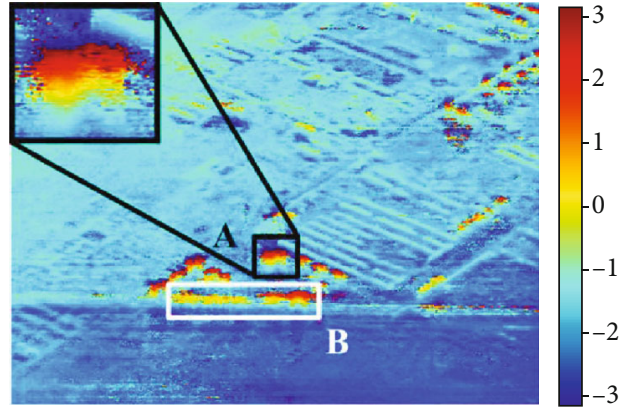

(b)

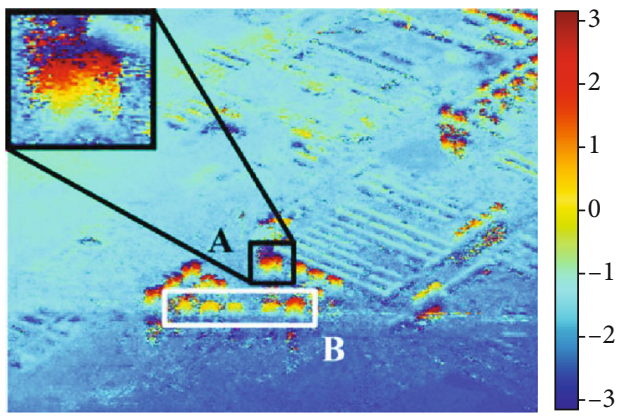

(d)

FIGURE 13: Interferograms of Scene in Figure 7(a). (a) Stripmap mode. (b) ScanSAR data processed by full-aperture approach. (c) ScanSAR data processed by the IPM-AIT. (d) ScanSAR data processed by the MIAA-AIT.

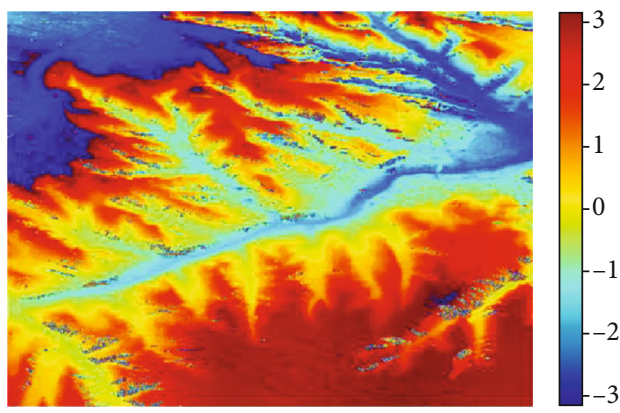

(a)

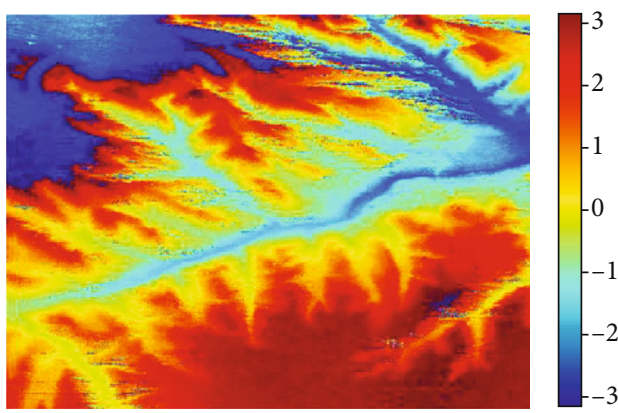

(c)

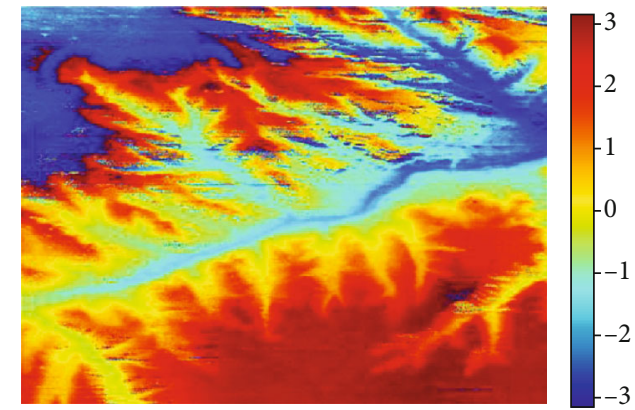

(b)

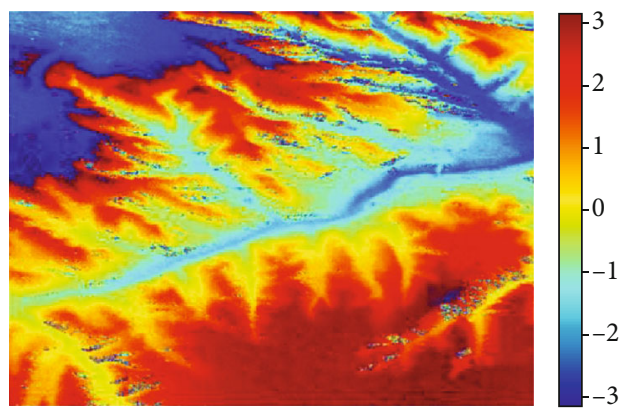

(d)

FIGURE 14: Interferograms of Scene in Figure 7(b). (a) Stripmap mode. (b) ScanSAR data processed by a full-aperture approach. (c) ScanSAR data processed by the LPM-AIT. (d) ScanSAR data processed by the MIAA-AIT.

simulation experiments, as shown in Figure 5, we have shown that the missing data recovered by the proposed algorithm has better quality than the missing data recovered by the
LPM-AIT. Due to the complexity of real SAR data, it is meaningless to directly compare the recovered data. Therefore, we can verify the effectiveness of the recovered data by 


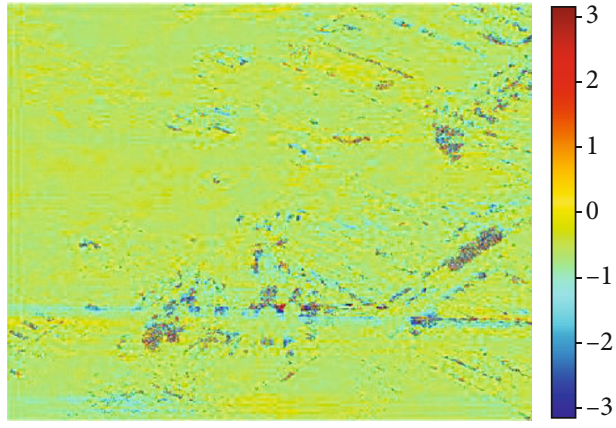

(a)

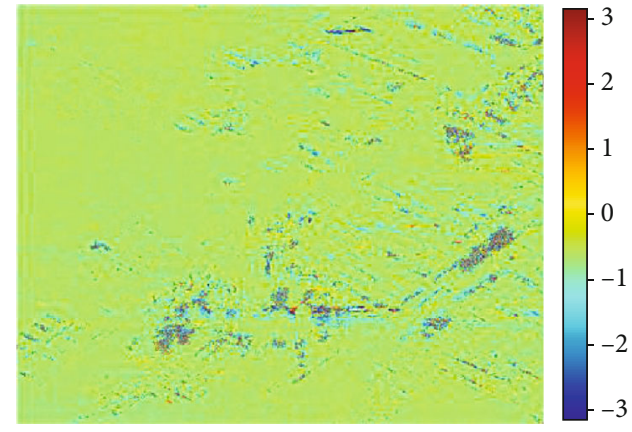

(b)

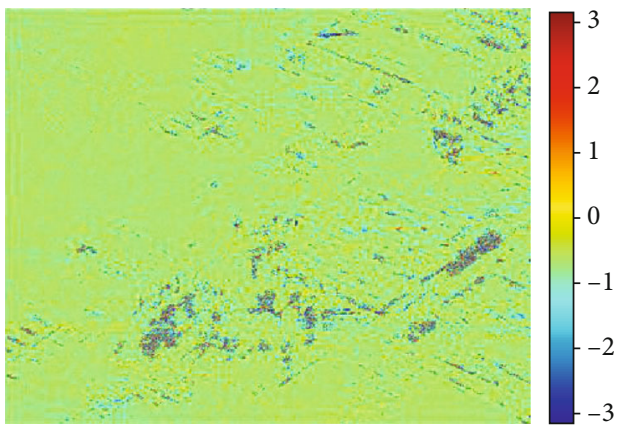

(c)

FIGURE 15: Difference maps of interferograms in Figure 13 processed by full-aperture, LPM-AIT, and MIAA-AIT, against Stripmap mode. (a) full-aperture against Stripmap; (b) LPM-AIT against Stripmap; (c) MIAA-AIT against Stripmap.

comparing the imaging results using only the recovered data. To compare the validity of the recovered data, we replace the available data (the burst) with zero and complete the imaging process using only the predicted data by LPMAIT and MIAA-AIT method, respectively. The imaging results are given in Figure 10. Obviously, the MIAA-AIT retains more details of the scene. The local scene marked by the yellow rectangle is enlarged and displayed in the lower right corner. We can see that in Figure 10(b) many relatively weak targets such as flat ground and low-rise houses are clearer than that in Figure 10(a). It shows that the signals of these relatively weak targets are not effectively recovered by the LPM-AIT, but can be better recovered by the proposed algorithm. More useful information can be remained in the data predicted by the proposed algorithm, so the final image processed by the proposed algorithm is better than that processed by the LPM-AIT.

In addition to an urban area, the results of the proposed algorithm applied to a rural area are presented in Figure 7(b). The results of the local scene marked by the red rectangle in Figure 7(b) are shown in Figure 11. The proposed algorithm also has a better performance in suppressing spikes in rural areas. Compared with Figure 11(b) and 11(c), the shadows are more properly defined, and the contours of the mountains are more distinguishable in Figure 11(d). The spikes neighboring the strong scatterers are almost all eliminated in Figure 11(d). Azimuth profiles of the point-like target marked by a red circle in Figure 11(a) are plotted in Figure 12, where a green line, a red line, and a blue line represent the azimuth profiles using the full-aperture algo-

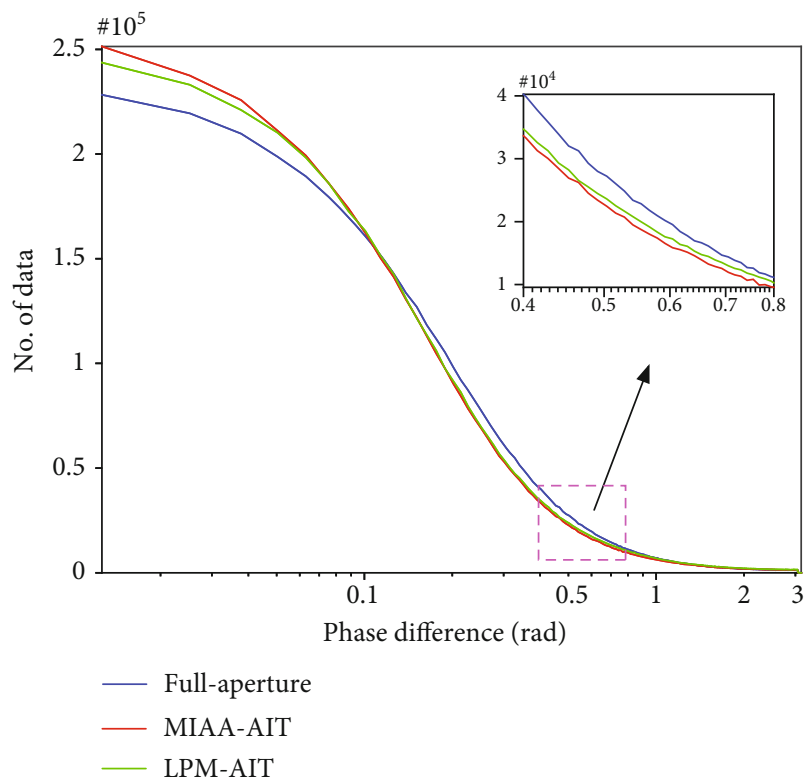

FIgURE 16: Statistical curve of the absolute value of the phase difference shown in Figure 15.

rithm, the LPM-AIT, and the MIAA-AIT, respectively. It can be seen that spikes are more thoroughly eliminated by the proposed algorithm, and the azimuth resolution of the target significantly increased compared with the result processed by the full-aperture approach. 
TABLE 2: Statistical results of difference maps of interferograms processed by full-aperture, LPM-AIT, and MIAA-AIT, against Stripmap mode. (a) Interferograms in Figure 12. (b) Interferograms in Figure 13.

(a)

\begin{tabular}{lccc}
\hline & Phase diff. $<0.1 \mathrm{rad}$ & $0.1 \mathrm{rad} \leq$ phase diff. $\leq 0.5 \mathrm{rad}$ & Phase diff. $>0.5 \mathrm{rad}$ \\
\hline Full-aperture & $49.49 \%$ & $35.84 \%$ & $14.67 \%$ \\
LPM-AIT & $52.37 \%$ & $33.29 \%$ & $14.34 \%$ \\
MIAA-AIT & $55.08 \%$ & $31.83 \%$ & $13.09 \%$ \\
\hline
\end{tabular}

(b)

\begin{tabular}{lccc}
\hline & Phase diff. $<0.1 \mathrm{rad}$ & $0.1 \mathrm{rad} \leq$ phase diff. $\leq 0.5 \mathrm{rad}$ & Phase diff. $>0.5 \mathrm{rad}$ \\
\hline Full-aperture & $43.44 \%$ & $41.56 \%$ & $15.00 \%$ \\
LPM-AIT & $44.83 \%$ & $42.41 \%$ & $12.76 \%$ \\
MIAA-AIT & $45.96 \%$ & $44.24 \%$ & $9.80 \%$ \\
\hline
\end{tabular}

Image contrast (IC) value can be used to evaluate the quality of the SAR image [32]. Here, we use the standard deviation amplitude contrast function in reference [32] to calculate the IC value of each image as shown in (9).

$$
\mathrm{IC}=\frac{\sqrt{E\left\{(A-E\{A\})^{2}\right\}}}{E\{A\}} \text {, }
$$

where $A$ is the amplitude of the image, $E\{\bullet\}$ means averaging operation, thus $\mathrm{E}\{\mathrm{A}\}$ is the mean of the image amplitude. Table 1 shows the IC value comparison of the imaging results by three different methods on the urban scene shown in Figure 8, and the nonurban sensen shown in Figure 11. The IC value of the image processed by the proposed algorithm is higher than that processed by the LPM-AIT in both two scenes, which means the image quality obtained by the proposed method is better. This further illustrates the effectiveness of the processed algorithm.

4.2.2. The Results of Interferometry Application. Section 4.2.1 has thoroughly demonstrated the spike suppression ability of the proposed algorithm over the ScanSAR images. Interferometric application can also benefit from the remarkable spike suppression performance to improve the ScanSAR interferogram quality. In this section, interferograms obtained using the proposed algorithm are presented to show the ability of the method in interferometry application. Interferograms of the two representative scenes in Figure 7 processed using the Stripmap mode, the full-aperture approach, the LPMAIT, and the proposed approach are shown in Figures 13 and 14.

Interferograms are contaminated due to phase information in the spikes when they are processed with only the gapped data, as shown in Figure 13(b). Sites A and B in Figure 13 are buildings. The shape of the building at site $\mathrm{A}$ processed by the MIAA-AIT is narrower than that processed by the LPM-AIT and more similar to that processed by the Stripmap mode. In site B, although it has been improved compared to Figure 13(b), the outlines of the buildings proc- essed by the LPM-AIT is blurred due to the phase information in spikes. The outlines of the buildings processed by the MIAA-AIT are clearer than the LPM-AIT and more similar to that processed by the Stripmap mode. Figure 15 shows three-phase difference maps of interferograms in Figure 13 processed by full-aperture, LPM-AIT, and MIAA-AIT, against Stripmap mode. It can be seen that on both sides of buildings, the phase difference of MIAA-AIT is much smaller than the LPM-AIT. Figure 16 shows the statistical curve of the absolute value of the phase difference. To show the results more clearly, the phase difference is displayed in logarithmic format. And the display range is only to $\pi$, because the differences are almost within $\pi$. Table 2 (a) shows the numerical statistics results. It can be seen that the point number of MIAA-AIT with very small phase difference are larger than that of the LPM-AIT and full-aperture. And the point number of MIAA-AIT with large phase difference is smaller than that of the full-aperture and LPM-AIT. Therefore, we can conclude the MIAA-AIT has better spike performance in interferometric application in urban areas.

In the rural scene, the interferogram processed by the proposed algorithm also has good performance in spike suppression, as shown in Figure 14. The interferogram processed by the full-aperture approach exists spikes, introducing additional noise to the interferogram. It can be seen that the spike phenomenon in the interferogram processed by the MIAAAIT is less than that of LPM-AIT, but it is not so obvious. The reason is that there are fewer bright points in the rural area than in the urban area. Figure 17 shows three-phase difference maps of interferograms in Figure 14 processed by full-aperture, LPM-AIT, and MIAA-AIT, against Stripmap mode. The corresponding statistical results are shown in Figure 18. Same as Figure 16, the phase difference is displayed in logarithmic format and the display range is only to $\pi$. Table 2(b) shows the numerical statistics. In the range where the phase difference is close to 0 , the point number of MIAAAIT is much higher than the number of full-aperture and LPM-AIT. And the point number of the MIAA-AIT with large phase difference is smaller than that of full-aperture and LPM-AIT. Therefore, it can be concluded that the effect 


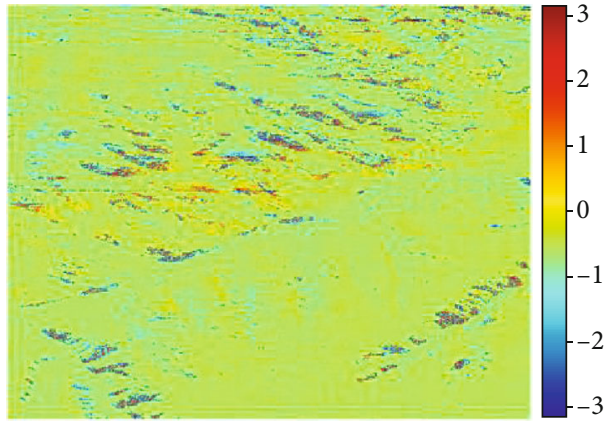

(a)

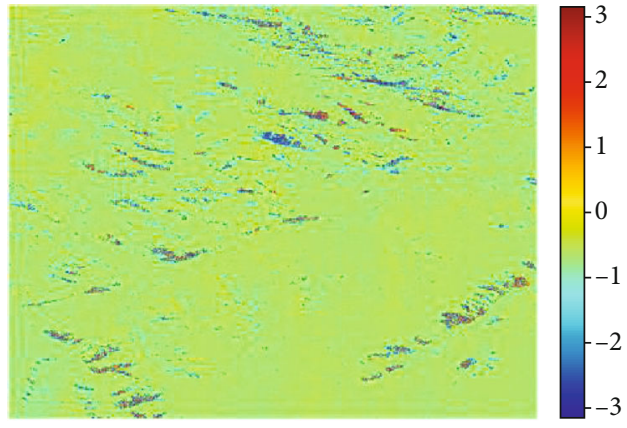

(b)

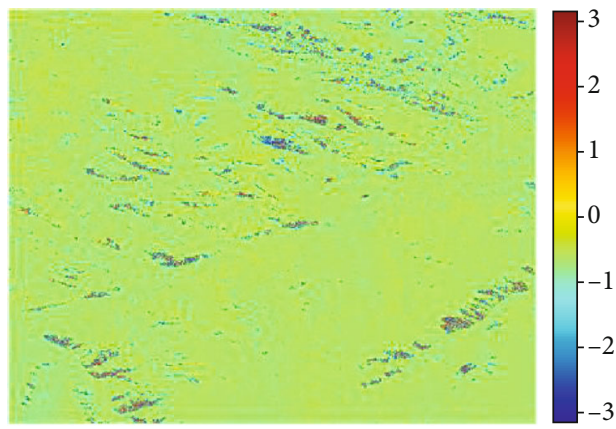

(c)

FIGURE 17: Difference maps of interferograms in Figure 14 processed by full-aperture, LPM-AIT, and MIAA-AIT, against Stripmap mode. (a) Full-aperture against Stripmap; (b) LPM-AIT against Stripmap; (c) MIAA-AIT against Stripmap.

of MIAA-AIT in rural area is still better than that of LPMAIT, but compared with urban areas, the advantages are not so prominent.

Based on the above analysis, the proposed algorithm can be used in interferometry application to improve interferograms in the ScanSAR mode.

\section{Conclusions}

This manuscript studies the spike contamination problems of ScanSAR mode data. When ScanSAR data are processed using the full-aperture approach, images are contaminated by spikes, and additional noise is introduced to the interferograms. Spike contamination of full-aperture ScanSAR image limits ScanSAR-related applications, such as target detection and interferometry. To solve this problem, an improved algorithm named the MIAA-AIT is proposed in this manuscript. In the MIAA-AIT, the gaps between bursts are recovered by the MIAA, which is a nonparametric missing-data recovery methodology. Compared with the existing LPM based algorithm, the proposed algorithm has a better performance on spike suppression and can improve the azimuth resolution of the full-aperture ScanSAR images. Experiment results show that more useful information can be remained in the data predicted by the proposed algorithm, therefore the final image processed by the proposed algorithm is better than that processed by the existing LPM based algorithm. The proposed MIAA-AIT algorithm has better applicability than the LPM-AIT for spike suppression and azimuth resolution enhancement when processing

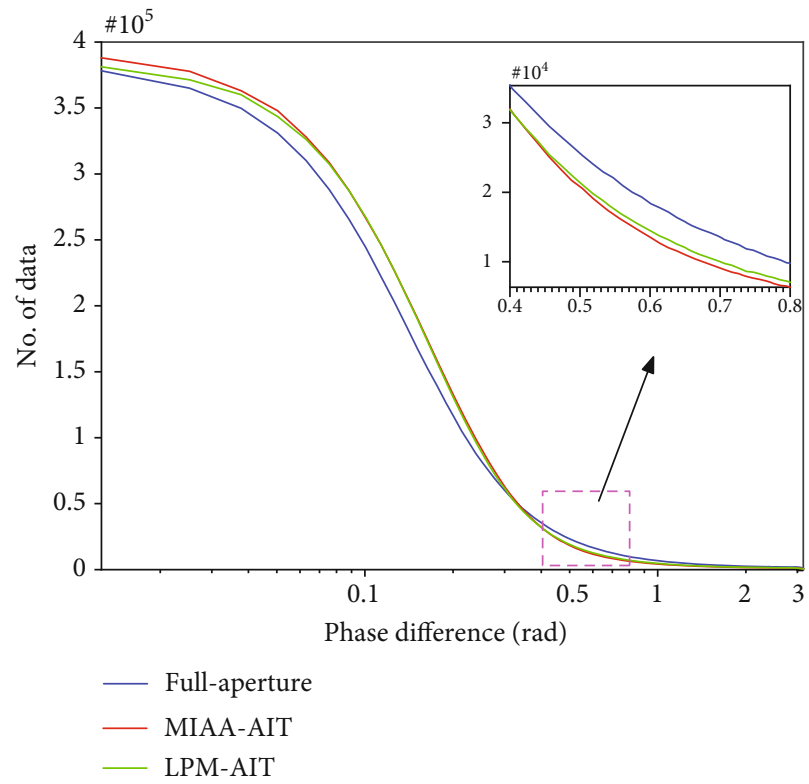

FIGURE 18: Statistical curve of the absolute value of the phase difference shown in Figure 16.

ScanSAR data by the full-aperture imaging algorithm. The proposed algorithm can also be used in interferometry to improve the quality of interferograms.

The results of this study show that by infilling the gaps between bursts with valid samples, the ScanSAR images processed by full-aperture approach can be significantly 
improved. The spikes can be suppressed effectively, and the azimuth resolution can be improved at the same time. This is very beneficial to ScanSAR-related applications. Therefore, it is worth further study.

\section{Data Availability}

The raw SAR data used to support the findings of this study have not been made available because the data used in this article is owned by the Department of Space Microwave Remote Sensing System, Aerospace Information Research Institute, Chinese Academy of Sciences, Beijing, China.

\section{Conflicts of Interest}

The authors declare no conflict of interest.

\section{Acknowledgments}

The authors would like to thank the anonymous reviewers for their constructive comments on an earlier version of this paper. This research was funded by the National Key R\&D Program of China grant number 2017YFB0502700.

\section{References}

[1] Y. Deng, F. Zhao, and R. Wang, "Brief analysis on the development and application of spaceborne SAR," Journal of Radars, vol. 17, pp. 1-10, 2012.

[2] Y. Deng and R. Wang, "Exploration of advanced bistatic SAR experiments," Journal of Radars, vol. 3, pp. 1-9, 2014.

[3] K. Tomiyasu, "Conceptual performance of a satellite borne, wide swath synthetic aperture radar," IEEE Transactions on Geoscience and Remote Sensing, vol. GE-19, no. 2, pp. 108116, 1981.

[4] R. K. Moore, J. P. Claassen, and Y. H. Lin, "Scanning spaceborne synthetic aperture radar with integrated radiometer," IEEE Transactions on Aerospace and Electronic Systems, vol. AES-17, no. 3, pp. 410-421, 1981.

[5] I. G. Cumming, Y. Guo, and F. Wong, Analysis and precision processing of RADARSAT ScanSAR data, Proc. Geomatics in the Era of RADARSAT; Publishing House, Ottawa, Canada, 1997.

[6] A. M. Guarnieri and C. Prati, "ScanSAR focusing and interferometry," IEEE Transactions on Geoscience and Remote Sensing, vol. 34, no. 4, pp. 1029-1038, 1996.

[7] J. Holzner and R. Bamler, "Burst-mode and ScanSAR interferometry," IEEE Transactions on Geoscience and Remote Sensing, vol. 40, no. 9, pp. 1917-1934, 2002.

[8] J. Ning, R. Wang, Y. Deng, N. Li, H. Song, and W. Fei, “AETIAA: a novel approach to enhance azimuth resolution for ScanSAR interferometry," EEE Journal of Selected Topics in Applied Earth Observations and Remote Sensing, vol. 10, no. 12, pp. 5674-5685, 2017.

[9] A. Moreira, J. Mittermayer, and R. Scheiber, "Extended chirp scaling algorithm for air- and spaceborne SAR data processing in stripmap and ScanSAR imaging modes," IEEE Transactions on Geoscience and Remote Sensing, vol. 34, no. 5, pp. 11231136, 1996.
[10] R. Bamler and M. Eineder, "ScanSAR processing using standard high precision SAR algorithms," IEEE Transactions on Geoscience and Remote Sensing, vol. 34, no. 1, pp. 212-218, 1996.

[11] C. Liang, Q. Zeng, and J. Jiao, "An assessment of ScanSAR interferometric processing using full-aperture approach," IEEE Geoscience and Remote Sensing Letters, vol. 11, no. 9, pp. 1559-1563, 2014.

[12] I. G. Cumming and F. H. Wong, Digital Processing of Synthetic Aperture Radar Data: Algorithms and Implementation, Artech House, Norwood, MA, USA, 2005.

[13] C. Liang, Z. Liu, E. J. Fielding, and R. Bürgmann, "InSAR time series analysis of L-Band wide-swath SAR data acquired by ALOS-2," IEEE Transactions on Geoscience and Remote Sensing, vol. 56, no. 8, pp. 4492-4506, 2018.

[14] N. Cao, H. Lee, H. C. Jung, and H. Yu, "Estimation of water level changes of large-scale Amazon wetlands using ALOS-2 ScanSAR differential interferometry," Remote Sensing, vol. 10, no. 6, pp. 966-981, 2018.

[15] G. Gomba, F. R. González, and F. De Zan, "Ionospheric phase screen compensation for the Sentinel-1 TOPS and ALOS-2 ScanSAR modes," IEEE Transactions on Geoscience and Remote Sensing, vol. 55, pp. 223-235, 2017.

[16] A. Pepe, A. B. Ortiz, P. R. Lundgren, P. A. Rosen, and R. Lanari, "The stripmap-ScanSAR SBAS approach to fill gaps in Stripmap deformation Time Series with ScanSAR data," IEEE Transactions on Geoscience and Remote Sensing, vol. 49, no. 12, pp. 4788-4804, 2011.

[17] R. Natsuaki, H. Nagai, T. Motohka et al., "SAR interferometry using ALOS-2 PALSAR-2 data for the Mw 7.8 Gorkha, Nepal earthquake," Earth, Planets and Space, vol. 68, no. 1, pp. 15-27, 2016.

[18] J. Horstmann, W. Koch, S. Lehner, and R. Tonboe, "Ocean winds from RADARSAT-1 ScanSAR," Canadian Journal of Remote Sensing, vol. 28, no. 3, pp. 524-533, 2014.

[19] M. Santoro, C. Beer, O. Cartus et al., "Retrieval of growing stock volume in boreal forest using hyper-temporal series of Envisat ASAR ScanSAR backscatter measurements," Remote Sensing of Environment, vol. 115, no. 2, pp. 490-507, 2011.

[20] A. S. Arnesena, T. S. F. Silva, L. L. Hess et al., "Monitoring flood extent in the lower Amazon River floodplain using ALOS/PALSAR ScanSAR images," Remote Sensing of Environment, vol. 130, pp. 51-61, 2013.

[21] B. Rabus, M. Eineder, A. Roth, and R. Bamler, "The shuttle radar topography mission-a new class of digital elevation models acquired by spaceborne radar," ISPRS Journal of Photogrammetry and Remote Sensing, vol. 57, no. 4, pp. 241-262, 2003.

[22] N. Li, R. Wang, Y. Deng et al., "Improved full-aperture ScanSAR imaging algorithm based on aperture interpolation," IEEE Geoscience and Remote Sensing Letters, vol. 12, no. 5, pp. 1101-1105, 2015.

[23] I. G. Cumming, Y. Guo, and F. Wong, "A comparison of phase-preserving algorithms for burst-mode SAR data processing," in GARSS'97. 1997 IEEE International Geoscience and Remote Sensing Symposium Proceedings. Remote Sensing - A Scientific Vision for Sustainable Development, pp. 731733, Singapore, 1997.

[24] T. G. Moore, B. W. Zuerndorfer, and E. C. Burt, "Enhanced imagery using spectral-estimation-based techniques," Lincoln Laboratory Journal, vol. 10, pp. 171-186, 1997. 
[25] J. Salzman, D. Akamine, R. Lefevre, and J. C. Kirk, "Interrupted synthetic aperture radar (SAR)," IEEE Aerospace and Electronic Systems Magazine, vol. 17, no. 5, pp. 33-39, 2002.

[26] C. Liang and E. J. Fielding, "Interferometric processing of ScanSAR data using stripmap processor: new insights from coregistration," IEEE Transactions on Geoscience and Remote Sensing, vol. 54, no. 7, pp. 4343-4354, 2016.

[27] R. Natsuaki, T. Motohka, M. Shimada, and S. Suzuki, "Burst misalignment evaluation for ALOS-2 PALSAR-2 ScanSARScanSAR interferometry," Remote Sensing, vol. 9, no. 3, pp. 216-224, 2017.

[28] T. Yardibi, J. Li, P. Stoica, M. Xue, and A. B. Baggeroer, "Source localization and sensing: a nonparametric iterative adaptive approach based on weighted least squares," IEEE Transactions on Aerospace and Electronic Systems, vol. 46, no. 1, pp. 425-443, 2010.

[29] P. Stoica, J. Li, and J. Lingi, "Missing data recovery via a nonparametric iterative adaptive approach," IEEE Signal Processing Letters, vol. 16, no. 4, pp. 241-244, 2009.

[30] M. Xue, L. Xu, and J. Li, "IAA spectral estimation: fast implementation using the Gohberg-Semencul factorization," IEEE Transactions on Signal Processing, vol. 59, no. 7, pp. 32513261, 2011.

[31] G.-O. Glentis and A. Jakobsson, "Efficient implementation of iterative adaptive approach spectral estimation techniques," IEEE Transactions on Signal Processing, vol. 59, no. 9, pp. 4154-4167, 2011.

[32] F. Berizzi and G. Corsini, "Autofocusing of inverse synthetic aperture radar images using contrast optimization," IEEE Transactions on Aerospace and Electronic Systems, vol. 32, no. 3, pp. 1185-1191, 1996. 